\title{
Devaluación del trabajo de posgraduados en México y migración internacional: los profesionistas en ciencia y tecnología
}

\author{
FERNANDO LOZANO ASCENCIO* \\ LUCIANA GANDINI** \\ TELÉSFORO RAMÍREZ-GARCÍA***
}

RESUMEN: El objetivo de este trabajo es comparar las condiciones de inserción laboral de los trabajadores mexicanos con posgrado tanto en México como en Estados Unidos con el fin de explorar la relación entre las condiciones del mercado laboral mexicano y la migración hacia Estados Unidos. El estudio analiza la correspondencia entre las credenciales académicas de los posgraduados mexicanos y el tipo de ocupación que desempeñan, en ambos países, con énfasis en aquellos formados en áreas de ciencias, tecnología, ingeniería y matemáticas (CTIM). Los resultados muestran que el mercado laboral mexicano "castiga» a los posgraduados en las áreas de CTIM, a los más jóvenes, y ofrece condiciones menos favorables para las mujeres y para los que poseen estudios de doctorado. En contraste, el mercado laboral de Estados Unidos "premia» a los posgraduados mexicanos en las áreas de cTim, a los más jóvenes, favorece a las mujeres, a los que cuentan con grado de doctor, a los que estudiaron en aquel país y a los mexicanos que poseen la ciudadanía estadounidense.

PALABRAS ClaVE: migración calificada, trabajo de posgraduados, México-Estados Unidos, abuso de cerebros, profesionistas de ciencias y tecnología.

*Investigador del Centro Regional de Investigaciones Multidisciplinarias (CRIM) de la UNAM, México.

**Investigadora del Instituto de Investigaciones Jurídicas de la UNAM, México.

***Investigador del Programa de Cátedras para Jóvenes Investigadores del Conacyt, adscrito al Centro Regional de Investigaciones Multidisciplinarias (CRIM) de la UNAM, México. 
ABSTRACT: The aim of this work is to compare the conditions of labor market integration of Mexican workers with post-graduate education in both Mexico and the United States, in order to explore the relationship between the Mexican labor market and migration to the United States. The study analyzes the relationship between the academic credentials held by Mexican post-graduates and the kind of employment they obtain, in both countries, with an emphasis on the sciences, technology, engineering and mathematic (CTIM) fields. The results indicate that the Mexican labor market "punishes» post-graduates in cTim fields, the younger jobseekers, and offers less-favorable conditions for women and for those who hold a $\mathrm{PhD}$. In contrast, the United States labor market «rewards» Mexican post-graduates in CTIM fields, those who are younger, women, those with a doctorate, those who studied in the United States, and those Mexicans who hold U.S. citizenship.

KEY WORDS: skilled migration, post-graduate employment, Mexico-United States, brain drain, science and technology professionals.

$62 \frac{\text { SEGUNDO SEMESTRE } 2015}{\text { MIGRACIÓN Y DESARROLLO NÚM. } 25}$ 


\section{INTRODUCCIÓN}

U

no de los aspectos que ha caracterizado a la migración de mexicanos hacia otros países durante las últimas dos décadas ha sido la creciente incorporación de personas con altos niveles de escolaridad a este torrente migratorio. La salida de población con estudios de posgrado (maestría, doctorado o alguna especialidad poslicenciatura) ha sido materia de debate en diversos sectores de la sociedad mexicana, la academia, las autoridades gubernamentales y los organismos internacionales que operan en México, no sólo por la decisión de muchos profesionistas de asentarse de forma definitiva en otro país, sino también por el hecho de que una parte muy significativa de los que salen a estudiar al extranjero con financiamiento gubernamental - específicamente del Consejo Nacional de Ciencia y Tecnología (Conacyt) - decide no regresar a México y buscar oportunidades en el extranjero.

Durante los últimos años, México ha experimentado un crecimiento histórico de su población con estudios de posgrado. De acuerdo con cifras oficiales, entre 2000 y 2010, el país casi triplicó su población de posgraduados al pasar de 355 mil a 921 mil personas. Frente a este inusitado crecimiento surgen las siguientes preguntas: $\grave{h}$ ha logrado este numeroso contingente de trabajadores altamente calificados incorporarse al mercado de trabajo en México y en qué condiciones lo ha hecho?, ża qué factores del ámbito laboral se puede atribuir el hecho de que esta población se sienta atraída por trabajar fuera de México?, żqué ventajas laborales representa para un posgraduado mexicano trabajar en el extranjero en vez de hacerlo en su país?

Para dar respuesta a las preguntas, este trabajo propone como hipótesis de trabajo que un factor central que motiva a los posgraduados mexicanos a buscar oportunidades fuera de México tiene que ver con la subutilización de las capacidades en el mercado laboral mexicano, característica que aquí también denominaremos como devaluación del trabajo o trabajo devaluado. En tal virtud, el trabajo devaluado de los profesionistas con posgrado en México es un aspecto que podría estar vinculado con la migración a otros 
países, situación que no los exime de que las condiciones de inserción laboral sean más desventajosas que en el país de origen. Este argumento nos llevó a proponer una estrategia analítica comparativa, esto es, comparar las condiciones de inserción laboral de los trabajadores mexicanos con posgrado tanto en el origen (México) como en el destino (Estados Unidos) con el fin de explorar si existe alguna relación entre las condiciones de mercado laboral mexicano y la migración hacia Estados Unidos. La variable empleada para analizar las condiciones de inserción laboral de los posgraduados mexicanos, tanto en México como en Estados Unidos, es la correspondencia entre sus credenciales académicas y el tipo de ocupación que desempeñan, esto es, si los posgraduados laboran en actividades en las que utilizan sus conocimientos y habilidades (ocupaciones de alta calificación) o en actividades en las que subutilizan sus capacidades (ocupaciones de media y escasa calificación).

Adicionalmente, nos propusimos concentrar el análisis en los profesionistas con posgrado en las áreas de ciencia, tecnología, ingeniería y matemáticas (CTIM), en virtud de que constituyen un sector fundamental en los procesos de innovación e incremento de la productividad, además de que su actividad está ligada a la generación de nuevas ideas, nuevas tecnologías, nuevas empresas e industrias. Ello explica el porqué, a nivel global, y más específicamente en los países industrializados, existe una política de formación y reclutamiento de profesionistas en estas áreas del conocimiento.

El trabajo consta de las siguientes partes: después de esta introducción, se presenta una breve revisión de los principales debates conceptuales, en particular sobre la noción de devaluación del trabajo calificado. En una siguiente sección se presentan los resultados del análisis comparativo de las condiciones laborales de los posgraduados mexicanos en стім tanto en México como en Estados Unidos para 2010. La última parte incluye una discusión final y las conclusiones del trabajo. 


\section{Devaluación del trabajo calificado}

El aspecto central que se propone discutir en este estudio es el referido a la subutilización o devaluación del trabajo de los mexicanos con posgrado, particularmente en áreas de CTIM en México y en Estados Unidos. Con ese fin, este apartado tiene la pretensión de ubicar cómo ha sido abordado este tema e identificar las principales perspectivas y conceptos que contribuyen a su explicación. La preocupación por el vínculo entre las habilidades adquiridas y aquéllas que son requeridas en el mercado de trabajo no es nueva, pero ha logrado impulso en la literatura reciente sobre la movilidad internacional de personas calificadas, caso específico en el cual se añaden otros elementos que podrían interferir en su propio proceso de incorporación social y laboral. Lo que interesa de manera específica en este trabajo es identificar e interpretar el fenómeno de la devaluación del trabajo calificado tanto en el origen como en el destino de manera integral.

La manifestación de asincronía entre capacidades adquiridas y requeridas ha tenido lugar a partir de la concurrencia de al menos tres hechos: el incremento de las instituciones de educación superior, el aumento de la matrícula y de los egresados con esos niveles de formación y la falta de desarrollo de la estructura ocupacional que dé cabida a contingentes mayores de personas con niveles educativos más altos. Este fenómeno, que inicialmente afectó a los países con mayores grados de industrialización, se ha extendido al resto del mundo en la medida en que prácticamente todos los países elevaron sus niveles promedio de educación formal. De tal forma, que sin ser nuevo, tampoco parece ser un hecho pasajero o coyuntural. Por el contrario, este desajuste sugiere haberse convertido en un fenómeno de carácter estructural.

El caso de los profesionistas con posgrado en áreas de cTim es en la actualidad uno de los grupos objetivo en el desarrollo de políticas de formación y reclutamiento a nivel global. Una adecuada comprensión del fenómeno requiere un análisis sobre cómo se encuentra México en términos de aprovechamiento de esta fuerza de trabajo, así como de la medida en que están siendo aprovechadas o no sus habilidades fuera del país. 
Desde la academia, las posturas clásicas elaboradas en torno a los conceptos de brain drain y bran gain se preocuparon más por quién se apropiaba de esas habilidades (el origen o el destino) que por el uso que se hacía de ellas. Por eso las conclusiones a las que se arriba con estas perspectivas se resumen en saldos de pérdidas o ganancias. Una tercera postura se centra alrededor del concepto de subutilización de habilidades. Tal noción alude precisamente al desajuste entre los requerimientos del empleo y el nivel de habilidades, fenómeno que algunos autores han denominado como brain waste, ya que lo interpretan como un desperdicio de habilidades o niveles de educación. Esta situación tiene lugar cuando las personas trabajan en ocupaciones cuyos requisitos de calificación son más bajos que su niveles de educación (Mattoo, Neagu y Özden, 2005). Desde la lógica precedente, en este caso en realidad sólo hay "perdedores» (los propios profesionistas migrantes y el país de origen) y no hay ganadores. Otra forma en que se ha analizado esta subutilización es como una expresión de subempleo, definido por aquellas situaciones en donde el nivel de empleo de las personas, en términos de la duración o de la productividad del trabajo, se sitúa por debajo de sus capacidades y preferencias (OIT, 1998).

Un conjunto de trabajos más recientes han abordado el tema a partir del concepto de de-skilling, ${ }^{1}$ el cual refiere a la pérdida de acceso a las ocupaciones que los profesionistas tenían previamente (en el caso de la población migrante, con antelación a la movilidad internacional). Conceptos asociados a esta propuesta analítica son los de job devaluation y downgrading occupational (mobility) en la medida en que dicha descualificación también es interpretada como una forma de devaluación o no reconocimiento de las credenciales educativas y una pérdida de estatus ocupacional y social o, en otras palabras, una degradación ocupacional. Bajo esta perspectiva se ha estudiado, por ejemplo, el caso de la descualificación que sufren las enfermeras - grupo por antonomasia en los estudios sobre migración calificada - al insertarse en puestos de trabajo doméstico de cuidados, lo que en la literatura se conoce como cadenas globales de cuidados (Salami y Nelson, 2014).

${ }^{1}$ Aunque el concepto tiene antecedentes en el planteamiento de Braverman (Wood, 1981).

$66 \frac{\text { SEGUNDO SEMESTRE } 2015}{\text { MIGRACIÓN Y DESARROLLO NÚM. } 25}$ 
El fenómeno de la descualificación puede ser visto de varias maneras (Siar, 2013): 1) como una etapa de transición que les toma a los inmigrantes adaptarse a las «normas» del país de acogida (Jasso, Rosenzweig y Smith, 2002); 2) como una forma de llenar la escasez de mano de obra en el mercado secundario por la explotación del trabajo de enclave económico de un país receptor (Piore, 1979), o3) como una forma de discriminación institucionalizada (Bauder, 2003).

Desde la primera aproximación, se trataría de un efecto temporal en la medida en que el supuesto que está por detrás es que las habilidades de las personas migrantes no son perfectamente transferibles al trasladarse de un país a otro, al menos inicialmente (Jasso, Rosenzweig y Smith, 2002), por eso se analiza a partir de la noción de transferibilidad del capital humano, inicialmente planteada por Becker (1964). La mayor limitante de este planteamiento es precisamente que observa al fenómeno como transitorio. Basta una revisión de la literatura para observar que aun con más de 10 años de tiempo de estancia, una porción importante de migrantes calificados no logra una inserción adecuada (Lozano y Gandini, 2010).

La segunda aproximación, planteada por Michael Piore (1979), parte de la concepción de mercado laboral segmentado - particularmente en los países industrializados-, según la cual el primario de uso intensivo de capital es altamente calificado, ofrece mejores condiciones salariales y laborales, y está reservado para la población nativa. El mercado secundario, de puestos menos calificados, peor remunerados y estatus más bajo es satisfecho por la población extranjera. Los migrantes, incluso los más calificados, están dispuestos a aceptar esas ocupaciones, ya que los concibe como migrantes temporales. El problema surge cuando, con el tiempo, ello no ocurre, los migrantes calificados permanecen, adquieren habilidades y experiencia, y entran en competencia con la población nativa. Se produce un vacío del mercado secundario que genera la repetición del ciclo y, consecuentemente, una continua dependencia de mano de obra migrante. Así, la descualificación sucede porque existe un mercado secundario de menor paga y más bajo estatus que los migrantes están dispuestos a aceptar. Este 
modelo analítico trata a la descalificación como una decisión racional y consciente, tomada por los migrantes para entrar en el mercado de trabajo en el extranjero. Su simplicidad hace ineficaz la comprensión del fenómeno de la descualificación más allá de la lente económica (Siar, 2013).

Finalmente, la tercera aproximación, postulada por Bauder (2003), entiende que la descualificación o devaluación del trabajo es una estrategia deliberada de los países de acogida para preservar el orden social. No se trata de un estado temporal, transicional, sino que la descualificación es una acción sistemática en donde las instituciones reguladoras —entre ellas el propio mercado laboral - excluyen a las personas migrantes de los segmentos superiores del mercado de trabajo, principalmente debido al no reconocimiento de títulos extranjeros y a la desestimación de la experiencia laboral extranjera. Así, el lugar donde se llevaron a cabo los estudios se convierte en un mecanismo de diferenciación en el mercado laboral.

Para sustentar esta visión, Bauder (2003) recurre al concepto de capital cultural institucionalizado propuesto por Bourdieu (1986) para explicar cómo funciona la reproducción social dentro de una sociedad compuesta por los trabajadores nativos y migrantes. Le permite concluir que el capital cultural institucionalizado de los migrantes altamente calificados (grado de educación y formación) es un recurso de poder para entrar en un país de inmigración. ${ }^{2}$ La cualificación y la formación educativa de los migrantes calificados deberían ser capaces por sí mismas de colocarlos en un plano de igualdad con la población nativa, dado que poseen cualidades similares en términos de educación y formación, independientemente de su sexo, raza y etnia. El fenómeno de la descualificación niega este supuesto del capital humano con la devaluación del capital cultural de los migrantes calificados en el país anfitrión. Es decir, el valor de un capital es específico para un lugar o contexto.

En síntesis, desde una perspectiva empresarial, los inmigrantes descualificados representan la fuerza laboral «flexible». Ellos poseen niveles

${ }^{2}$ La teoría del capital humano ha hecho hincapié en cómo las inversiones en la educación podrían conducir a un aumento del bienestar personal (Becker, 1964).

$68 \frac{\text { SEGUNDO SEMESTRE } 2015}{\text { MIGRACIÓN Y DESARROLLO NÚM. } 25}$ 
de escolaridad altos, pero aceptan trabajar por salarios bajos o en ocupaciones que no se corresponden con su educación formal. Desde una perspectiva de capital humano, sin embargo, la descualificación es una pérdida tanto para el país de destino como para el país de origen (Bauder, 2003). Esta propuesta analítica permite comprender la complejidad del fenómeno al evidenciar que no hay una adecuada utilización en ninguno de los contextos.

Subutilización en el destino: iy en el origen?

De la revisión conceptual previa surge una primera apreciación: la literatura sobre migración calificada se ha centrado exclusivamente en lo que ocurre en los mercados laborales receptores pertenecientes a países del Norte global. En particular los desarrollos teóricos y empíricos sobre descualificación y devaluación han acentuado el potencial desaprovechamiento de los recursos humanos en esos contextos, pero poco han reflexionado respecto a esta situación en los contextos de origen y qué consecuencias tiene este «desperdicio» para la propia población migrante.

Una mirada analítica de este último tipo permite identificar la existencia de una doble paradoja. Por un lado, cuando los países, como es el caso de México, que «pierden» trabajadores con posgrados en áreas de CтIM se preocupan por su retención o recuperación, pero esa masa crítica experimenta enormes dificultades para el acceso a un empleo digno en sus países de origen y en correspondencia con sus calificaciones, en la medida en que el mercado no ofrece adecuadas oportunidades de aprovechamiento de sus habilidades. Por el otro, los países que "ganan» trabajadores en áreas de CTIM, como el caso estadounidense, que además implementan políticas explícitas de reclutamiento y favorecimiento de la migración de esta fuerza de trabajo específica, tampoco garantizan la utilización de dichas capacidades. Es decir, aunque hayan admitido profesionales con base en las políticas de inmigración de los países de destino, muchos de ellos son relegados a una condición ocupacional inferior, de menores salarios, debido a la falta de reconocimiento 
de sus credenciales extranjeras y el sesgo que produce no haber adquirido su formación en el país de acogida, la falta de experiencia local, entre otros (Sial, 2013). Sus credenciales y habilidades en el extranjero con frecuencia no permiten obtener las recompensas laborales esperadas y los beneficios del desarrollo profesional que han sido una parte importante de su motivación para emigrar al extranjero, especialmente a los países más desarrollados.

Son precisamente estas situaciones contradictorias las que han llevado a proponer el concepto de «abuso de cerebros» (brain abuse) para su explicación: es un abuso del cerebro, porque no hay una adecuada utilización de las habilidades de las personas en ningún lado (Bauder, 2003). Adicionalmente, es necesario insistir en que las consecuencias que este desaprovechamiento produce no sólo deben ser vistas a nivel de los Estados receptores y emisores (sus mercados y sus sociedades) sino, y principalmente, en las propias personas calificadas.

Para finalizar esta sección quisiéramos enfatizar que de aquí se desprende la necesidad de promover estudios de corte comparativo que permitan comprender de manera simultánea cómo se expresa el proceso de devaluación del trabajo calificado en los mercados laborales contemporáneos del Norte y Sur globales. Algunas investigaciones se han aproximado a este fenómeno a partir de un análisis en términos de oferta y demanda, y lo explican por la existencia de una sobreoferta (en el origen) y un déficit en ciertos empleos (en los destinos), fundado en requerimientos demográficos (Nowicka, 2013). Sin embargo, hemos constatado en varios trabajos - y en el presente, como se verá más adelante- que el problema es de otro tipo. En el origen, no es la falta de acceso al empleo sino la inserción en puestos que promueven la subutilización de sus capacidades (Fernandez y Ortega, 2008; Hernández Laos, Solís y Stefanovich, 2013) y, en el destino, más que una escasez de mano de obra para cubrir ciertos espacios laborales, como el de aquellos que requieren trabajadores formados en áreas de CTIM, se trata de una política deliberada no sustentada en la demanda del mercado, sino en la posibilidad de atraer fuerza de trabajo extranjera calificada que está dispuesta a ocupar los puestos laborales que no toma la población nativa (Lozano y Ramírez, en prensa), presumiblemente en condiciones más precarizadas. 
En este sentido, queda claro entonces que el mercado laboral de origen también subutiliza las capacidades de la población con posgrado al exigir dichas credenciales educativas para ocupaciones que no las requieren o no las utilizan adecuadamente. La implementación de políticas de formación de recursos humanos posiblemente más credencialistas que sustantivas (Gandini y Lozano, 2012), la existencia de modelos de acumulación no acordes con dichas trasformaciones, así como la asincronía de los cambios en los niveles de educación de la fuerza de trabajo con la estructura ocupacional son algunas de las posibles explicaciones a este fenómeno. El siguiente apartado pretende dar luz al entendimiento de la devaluación del trabajo de los mexicanos con posgrado en áreas de CTIM en México y en Estados Unidos.

\section{ANÁlisis COMPARATIVO DE LAS CONDICIONES LABORALES DE LOS POSGRADUADOS MEXICANOS EN LOS MERCADOS DE TRABAJO de México y Estados Unidos, 2010}

En el presente trabajo proponemos una tipología de ocupaciones que permite identificar si existe una correspondencia entre el grado académico de los profesionistas y el tipo de ocupación que desempeñan. Ello permite estimar el porcentaje de posgraduados que laboran en ocupaciones en las que hacen uso de sus conocimientos y habilidades, y aquellas donde subutilizan sus credenciales y su formación profesional. Con base en la metodología propuesta por Hernández Laos, Solís y Stefanovich (2013) se determinaron dos tipos de ocupaciones: 1) las altamente calificadas, que supone una utilización de las capacidades de los posgraduados, y 2) las de mediana y escasa calificación, que implica una subutilización de las capacidades de los posgraduados, es decir, ocupaciones que podrían desempeñar trabajadores con licenciatura o con algún nivel menor de escolaridad. ${ }^{3}$ Mediante el uso de

${ }^{3}$ Para mayor detalle de la construcción de la tipología de ocupación, véase Hernández Laos, Solís y Stefanovich (2013). 
esta tipología, el cuadro 1 muestra que entre 2000 y 2010 se registró en México una disminución en el porcentaje de trabajadores con posgrado en ocupaciones altamente calificadas de 77.0 a 73.6 por ciento, tendencia que fue más acentuada en el caso de los hombres. En contraparte, se aprecia un incremento en el porcentaje de profesionistas con posgrado en ocupaciones de mediana o escasa calificación (23.0 por ciento en 2000 a 26.4 por ciento en 2010). Este último dato apunta a que en México existe una tendencia creciente, al menos durante la década 2000-2010, a la subutilización o devaluación de las capacidades de la población con estudios de posgrado.

Proponemos también clasificar al sector de trabajadores mexicanos con posgrado en dos grandes grupos: 1) aquellos que se formaron en áreas de CTIM, y 2) aquellos formados en otras áreas de conocimiento (no CTIM). Así, vemos que al relacionar la variable tipo de ocupación (altamente calificada vs. media y escasa calificación) con los dos grandes grupos enunciados (CTIM y no CTIM), observamos que entre 1990 y 2010 los trabajadores con posgrado en áreas de CTIM participan en una proporción menor en ocupaciones de alta calificación que los que se formaron en otras áreas del conocimiento (véase cuadro 1). Pese a que la diferencia en 2010 es menor que en los dos anteriores, este resultado muestra que en México existe una desventaja significativa para quienes se formaron en áreas de CTIM, al menos en lo que se refiere a ocupar puestos de alta calificación.

En virtud de que el propósito de este artículo es establecer si existe algún vínculo entre las condiciones laborales de los posgraduados en México y la migración de esta población a Estados Unidos, en lo que sigue de este apartado centraremos el análisis exclusivamente en 2010 y examinaremos las condiciones laborales de los posgraduados nacidos en México integrados tanto en el mercado de trabajo mexicano como en el estadounidense.

Las fuentes de información empleadas son el Censo de Población y Vivienda de 2010 y la American Community Survey (ACS) del trienio 2009-2011. Ambas fuentes, aunque tienen objetivos, conceptos y metodologías diferentes, permiten dimensionar el volumen de la población mexicana con estudios 


\section{CUADRO 1}

Población de 20 años o más con estudios de posgrado, nacida en México, según tipo de ocupación y área del conocimiento, 1990, 2000 y 2010

\begin{tabular}{|c|c|c|c|c|c|c|}
\hline \multirow[b]{2}{*}{ TIPO DE OCUPACIÓN } & \multicolumn{2}{|c|}{1990} & \multicolumn{2}{|c|}{2000} & \multicolumn{2}{|c|}{2010} \\
\hline & ABS. & $\%$ & ABS. & $\%$ & ABS. & $\%$ \\
\hline Población total & 209,670 & 100.0 & 277,805 & 100.0 & 678,949 & 100.0 \\
\hline Ocupaciones altamente calificadas & 161,340 & 76.9 & 214,008 & 77.0 & 499,675 & 73.6 \\
\hline $\begin{array}{l}\text { Ocupaciones de media y escasa } \\
\text { calificación }\end{array}$ & 48,330 & 23.1 & 63,797 & 23.0 & 179,274 & 26.4 \\
\hline СТIM & 53,160 & 100.0 & 53,253 & 100.0 & 157,576 & 100.0 \\
\hline Ocupaciones altamente calificadas & 37,460 & 70.5 & 37,217 & 69.9 & 110,530 & 70.1 \\
\hline $\begin{array}{l}\text { Ocupaciones de media y escasa } \\
\text { calificación }\end{array}$ & 15,700 & 29.5 & 16,036 & 30.1 & 47,046 & 29.9 \\
\hline No CTIM & 156,510 & 100.0 & 224,552 & 100.0 & 521,373 & 100.0 \\
\hline Ocupaciones altamente calificadas & 123,880 & 79.2 & 176,791 & 78.7 & 389,145 & 74.6 \\
\hline $\begin{array}{l}\text { Ocupaciones de media y escasa } \\
\text { calificación }\end{array}$ & 32,630 & 20.8 & 47,761 & 21.3 & 132,228 & 25.4 \\
\hline
\end{tabular}

Fuente: Estimaciones de los autores con base en INEGI: muestras censales: 1990, 2000 y 2010.

de posgrado residente en México y Estados Unidos, construir su perfil sociodemográfico y analizar sus condiciones laborales.

En el caso de la población con estudios de posgrado residente en México, la muestra censal fue de 46,928 casos que, expandidos, representan una población de 920,809 personas de 20 años o más nacidas en México y con al menos un año de estudios de posgrado. De ellos, 784,445 declararon que se encontraban trabajando o realizaban alguna actividad económica al momento de la entrevista censal, de las que se seleccionó un universo de 678,949 personas que contaban con la información requerida. ${ }^{4}$

Para los posgraduados mexicanos que viven en Estados Unidos, el tamaño de la muestra obtenido a partir de la base trianual de la ACs (20092011) fue de 4,043 casos que, al expandirla, representa una población de 143,338 personas. De ellas, 108,648 estaban empleadas, quienes constituyen

${ }^{4}$ Es decir, aquellas que además de estar ocupadas cumplen con el criterio de haber especificado el tipo de ocupación en que se empleaban y área del conocimiento en la que se formaron (34,598 casos sin ponderar). 
la población objetivo para análisis laboral de los posgraduados mexicanos en Estados Unidos. ${ }^{5}$

La estrategia metodológica consistió en realizar, primeramente, un análisis descriptivo de las características sociodemográficas de la población ocupada nacida en México de 20 años o más con estudios de posgrado, según nivel de calificación de la ocupación desempeñada. Posteriormente, se ajustó un modelo de regresión logística binomial con el fin de identificar los factores asociados a la probabilidad de que un profesionista con estudios de posgrado se inserte en una ocupación altamente calificada, es decir, de emplearse en una ocupación acorde con su grado y nivel de formación académica. ${ }^{6}$

Específicamente, se estimaron dos modelos logísticos: uno para la población residente en México y otro para los radicados en Estados Unidos. En ambos casos, el modelo tuvo como variable dependiente el nivel de calificación de la ocupación realizada. Se trata de una variable dicotómica que toma el valor de 1 si la persona se emplea en una ocupación altamente calificada o de 0 si lo hace en una ocupación de mediana o escasa calificación.

Se incluyeron siete variables independientes o predictores: 1) el sexo de la persona (hombre o mujer); 2) el grupo de edad, que comprende dos subgrupos: las personas menores de 40 años y las de esa edad o más; 3) la situación conyugal, que también divide a la población en dos subgrupos: unidos y no unidos conyugalmente; 4) el nivel de escolaridad, que abarca

${ }^{5}$ En el caso de la ACS, el tamaño de la muestra de la población ocupada sin ponderar fue de 3,036 , definida con los mismos criterios que la muestra de la población con posgrado residente en México.

${ }^{6}$ En un modelo logístico, la variable dependiente es dicotómica y toma el valor de 1 si el evento sucede y de 0 si no sucede. La estimación del modelo logístico se expresa matemáticamente de la siguiente forma: $n(p / 1-p)=\beta 0+\beta 1 X 1+\beta 2 X 2+\beta 3 X 3, \ldots, \beta p X p$. Dicha ecuación asume que la relación $\ln (p / 1-p)$ y las variables independientes $X 1, \ldots, X p$ es lineal. El término $(p / 1-p)$ se denomina razón de momios (Odds ratio, del término en inglés Odds) y representa la probabilidad de ocurrencia o no de la variable dependiente: $p(y=1) / p(y=0)$. La virtud de la regresión logística es que no sólo permite determinar el nivel de asociación entre la variable dependiente $(Y)$ y las variables independientes o predictores $(X i)$, sino que además permite estimar el peso específico de cada categoría, controlando mediante las demás variables incluidas en el modelo (Hosmer y Lemeshow, 1989).

$74 \frac{\text { SEGUNDO SEMESTRE } 2015}{\text { MIGRACIÓN Y DESARROLLO NÚM. } 25}$ 
tres subgrupos de profesionistas: personas con grado de maestría, profesional y doctorado; 5) tipo de carrera, la cual distingue a los posgraduados en las áreas de cTim de aquellos que se formaron en otros campos del conocimiento. Es decir, se divide en profesionistas CTIM y no CTIM, cuya clasificación fue tomada de Langdon y colaboradores (2011). Estas cinco variables explicativas fueron incluidas en los dos modelos logísticos enunciados. En el modelo estimado para los posgraduados residentes en Estados Unidos se incluyeron dos variables más: 6) el lugar de estudio, dentro o fuera de Estados Unidos (presumiblemente su país de origen), y 7) la condición de tenencia de la ciudadanía estadounidense, que clasifica a la población en personas ciudadanas y no ciudadanas estadounidenses.

El lugar de estudio es una variable proxi para determinar el país donde las personas recibieron su educación, ya sea en Estados Unidos o en México. Dicha variable fue construida a partir del año de llegada a Estados Unidos, el año de la encuesta y la edad de la persona al momento de la entrevista. A partir de la construcción de dicha variable se planteó la hipótesis de que la población que realizó sus estudios de posgrado en México corresponde al grupo de personas que ingresaron a Estados Unidos a los 21 años o más de edad, y que en ese momento ya contaban con al menos el nivel de licenciatura. Por su parte, los mexicanos educados en Estados Unidos se definen como las personas que cuentan con nivel de licenciatura o más, y que ingresaron a ese país antes de haber cumplido los 21 años de edad.

Análisis descriptivo de las variables incluidas en el modelo

El cuadro 2 contiene información sobre las características sociodemográficas de las personas de 20 años o más con estudios de posgrado residentes en México y Estados Unidos, según grado o nivel de especialización de la ocupación que desempeñan. En dicho cuadro se observa que mientras que en México 57.6 por ciento de los empleados en una ocupación altamente calificada son hombres, entre los que viven en Estados Unidos y desempeñan 
una ocupación similar, dicha cifra disminuye a 54 por ciento. Este resultado revela, además, que en este último país las mujeres mexicanas con posgrado tienen una mayor participación en ocupaciones altamente calificadas que sus connacionales que radican en México (42.4 por ciento versus 46 por ciento). $O$ dicho en otras palabras, las mujeres presentan una tendencia mayor a desempeñarse en un empleo ad hoc con su grado de formación académica o especialización laboral.

En cuanto a la edad promedio, de la información contenida en el cuadro 2 se desprende que, en general, las personas con posgrado en México son más jóvenes que las que viven en Estados Unidos: 41.5 y 43.1 años, respectivamente. Esta situación se presenta tanto entre los posgraduados que se emplean en los dos tipos de ocupaciones (de alta calificación y de media o escasa calificación); además, en ambas ocupaciones la proporción de personas menores de 40 años es menor entre los que residen en México que entre quienes lo hacen en Estados Unidos. Respecto a la situación conyugal de los posgraduados, se observa que en los residentes en ambos países, poco más de 6 de cada 10 personas estaban unidas o casadas al momento del censo o encuesta, y sólo alrededor de una tercera parte eran solteros, unidos o divorciados.

La distribución porcentual de la población por nivel de escolaridad de los posgraduados también muestra pequeñas diferencias según país de residencia y tipo de ocupación desempeñada. Así, por ejemplo, entre los que residen en México, 87 por ciento de los que cuentan con estudios de maestría y 13 por ciento de quienes tienen el grado de doctor se emplean en una ocupación altamente calificada. Ambos porcentajes son mayores que los registrados por sus pares radicados en Estados Unidos; sin embargo, es importante destacar que en este último país la proporción de maestros y doctores que se desempeñan en una ocupación mediana o escasamente calificada es menor que entre quienes viven en México, lo cual indica diferencias importantes en el nivel de subutilización de la mano de obra mexicana según país de residencia. 


\section{CUADRO 2}

Población ocupada nacida en México con estudios de posgrado por país de residencia y tipo de ocupación, según características seleccionadas, 2010 y 2009-2011

\begin{tabular}{|c|c|c|c|c|c|c|}
\hline \multirow[b]{3}{*}{$\begin{array}{l}\text { CARACTERÍSTICAS } \\
\text { SOCIODEMOGRÁFICAS }\end{array}$} & \multicolumn{3}{|c|}{ MÉXICO } & \multicolumn{3}{|c|}{ ESTADOS UNIDOS } \\
\hline & \multicolumn{3}{|c|}{ TIPO DE OCUPACIÓN } & \multicolumn{3}{|c|}{ TIPO DE OCUPACIÓN } \\
\hline & TOTAL & $\begin{array}{l}\text { ALTAMENTE } \\
\text { CALIFICADA }\end{array}$ & $\begin{array}{l}\text { MEDIANA O } \\
\text { ESCASAMENTE } \\
\text { CALIFICADA }\end{array}$ & TOTAL & $\begin{array}{l}\text { ALTAMENTE } \\
\text { CALIFICADA }\end{array}$ & $\begin{array}{l}\text { MEDIANA O } \\
\text { ESCASAMENTE } \\
\text { CALIFICADA }\end{array}$ \\
\hline Población total & 678,949 & 499,675 & 179,274 & 108,648 & 65,565 & 43,083 \\
\hline Sexo & 100.0 & 100.0 & 100.0 & 100.0 & 100.0 & 100.0 \\
\hline Hombre & 58.3 & 57.6 & 60.0 & 56.9 & 54.0 & 61.3 \\
\hline Mujer & 41.7 & 42.4 & 40.0 & 43.1 & 46.0 & 38.7 \\
\hline Grupos de edad & 100.0 & 100.0 & 100.0 & 100.0 & 100.0 & 100.0 \\
\hline Menor de 40 años & 47.6 & 46.1 & 51.9 & 41.4 & 43.6 & 38.0 \\
\hline 40 años o más & 52.4 & 53.9 & 48.1 & 58.6 & 56.4 & 62.0 \\
\hline Edad promedio (años) & 41.5 & 41.9 & 40.5 & 43.1 & 43.0 & 43.2 \\
\hline Estado civil & 100.0 & 100.0 & 100.0 & 100.0 & 100.0 & 100.0 \\
\hline Unido & 66.9 & 67.6 & 65.0 & 67.5 & 69.7 & 64.0 \\
\hline No unido & 33.1 & 32.4 & 35.0 & 32.5 & 30.3 & 36.0 \\
\hline Nivel de escolaridad & 100.0 & 100.0 & 100.0 & 100.0 & 100.0 & 100.0 \\
\hline Maestría & 87.8 & 86.6 & 91.2 & 64.7 & 66.7 & 61.7 \\
\hline Doctorado & 12.2 & 13.4 & 8.8 & 8.7 & 11.4 & 4.5 \\
\hline Profesional & - & - & - & 26.6 & 21.9 & 33.8 \\
\hline Tipo de carrera & 100.0 & 100.0 & 100.0 & 100.0 & 100.0 & 100.0 \\
\hline CTIM & 23.2 & 22.1 & 26.2 & 28.8 & 29.4 & 28.1 \\
\hline No cTim & 76.8 & 77.9 & 73.8 & 71.2 & 70.6 & 72.0 \\
\hline País de estudio & - & - & - & 100.0 & 100.0 & 100.0 \\
\hline Estados Unidos & - & - & - & 39.7 & 46.5 & 29.3 \\
\hline México & - & - & - & 60.3 & 53.5 & 70.7 \\
\hline Condición de ciudadanía & - & - & - & 100.0 & 100.0 & 100.0 \\
\hline Ciudadano estadounidense & - & - & - & 55.4 & 63.9 & 42.4 \\
\hline $\begin{array}{l}\text { No ciudadano } \\
\text { estadounidense }\end{array}$ & - & - & - & 44.6 & 36.1 & 57.6 \\
\hline
\end{tabular}

Fuente: Estimaciones de los autores con base en INEGI: Muestra del Censo de Población y Vivienda, 2010; e IPUMS: American Community Survey (ACS), 2009-2011.

En este mismo tenor, los datos muestran que la proporción de posgraduados mexicanos con una carrera en áreas de CTIM es mayor entre los radicados en Estados Unidos que los que viven en México: 28.8 y 23.2 por ciento, respectivamente. De hecho, cabe resaltar que el porcentaje de personas 
con posgrado en áreas de CTIM y que se emplean en ocupaciones altamente calificadas es mayor en los primeros que en los segundos (29.4 por ciento versus 22.1 por ciento), y por el contrario, el porcentaje de personas con posgrado en áreas de cTim laborando en ocupaciones mediana o escasamente calificadas es menor en los segundos que en los primeros (26.2 por ciento versus 28.2 por ciento). Estos primeros resultados muestran que el mercado de trabajo de Estados Unidos no sólo da más cabida a los posgraduados en áreas de CTIM, en comparación con el mercado laboral mexicano sino que emplea a más posgraduados en ocupaciones altamente calificadas.

En cuanto al país de obtención del grado de la población mexicana con posgrado inmigrante en Estados Unidos, se observa que 4 de cada 10 personas se graduaron en ese país. Entre los que se desempeñaban en una ocupación altamente calificada, el 53.5 por ciento estudió presumiblemente en México, proporción que se eleva a 70 por ciento entre quienes lo hacen en una ocupación que requiere un menor nivel de calificación. Asimismo, cabe destacar que más de la mitad de los posgraduados mexicanos que viven y trabajan en Estados Unidos cuenta con la ciudadanía estadounidense. Esta situación migratoria engloba a 63.9 por ciento de los que se emplean en una ocupación altamente calificada, pero sólo a 42.4 por ciento de los que lo hacen en ocupaciones de menor calificación. Estos resultados apuntan a que el país de estudios y el estatus migratorio son factores que inciden en la inserción laboral y en el tipo de empleos en los que se desempeña la población calificada mexicana radicada en aquel país.

Resultados del análisis multivariado:

factores asociados a la probabilidad de emplearse en una ocupación altamente calificada en México y en Estados Unidos

En este apartado se presentan los resultados de los modelos de regresión logística binaria estimados para el caso de México y Estados Unidos. En términos generales, podemos decir que los hallazgos destacados en el análisis descriptivo de las características sociodemográficas, educativas y migra-

$78 \frac{\text { SEGUNDO SEMESTRE } 2015}{\text { MIGRACIÓN Y DESARROLLO NÚM. } 25}$ 
torias con respecto al nivel de especialización de la ocupación en la que se emplea la población con posgrado se ven confirmados en los resultados de los modelos estimados, los cuales además nos ayudan a dimensionar el grado de influencia que ejercen las variables analizadas. De hecho, todas las variables independientes o predictores resultaron estadísticamente significativas a un 95 por ciento de confidencialidad $(p<0.05)$.

Con respecto al sexo, los resultados del modelo logístico estimado para la población con posgrado residente en México muestran que el hecho de ser mujer incrementa en casi 13 por ciento la propensión de insertarse en una ocupación altamente calificada en comparación con los hombres (categoría de referencia) (véase cuadro 3). Una situación similar se presenta entre la población mexicana con posgrado inmigrante en Estados Unidos, pues los odds ratio del modelo estimado también indican que las mujeres mexicanas con posgrado en Estados Unidos son 27 por ciento más propensas a insertarse en una ocupación altamente calificada en comparación con los hombres (véase cuadro 4). ${ }^{7}$ Estos resultados guardan relación con el considerable incremento en el nivel de escolaridad que la población femenina mexicana experimentó en las últimas dos décadas, así como con su cada vez mayor participación en la migración calificada mexicana que se dirige a Estados Unidos.

En cuanto a la edad, los datos también muestran diferencias importantes por grupos etarios y país de residencia. Para el caso de la población con posgrado residente en México, el modelo estimado indica que el tener menos de 40 años disminuye en 17 por ciento la propensión a emplearse en una ocupación altamente calificada en comparación con quienes tienen 40 años o más de edad. En cambio, entre la población con posgrado que vive en Estados Unidos se presenta una relación inversa, ya que la probabilidad de desempeñarse en una actividad altamente calificada es un 67 por ciento más alta entre las personas menores de 40 años que en aquellas que tienen 40 años o

7 La estrategia de exposición de los resultados de los modelos es la de comparar simultáneamente los resultados del modelo en México con los del modelo en Estados Unidos; por lo que cuando se haga referencia a las resultados del primero los datos corresponden al cuadro 3 , y cuando se analice el caso de Estados Unidos, al cuadro 4. 
más de edad. Estos resultados permiten suponer que las posibilidades para los jóvenes posgraduados mexicanos de emplearse en una ocupación acorde con su nivel de formación académica son más limitadas en México que en Estados Unidos, lo cual podría deberse a la incapacidad del mercado laboral mexicano para absorber en ocupaciones acordes a los profesionistas mexicanos que año con año egresan de las universidades y centros de investigación superior en el país.

Los resultados para la variable situación conyugal indican que las personas no unidas (conyugal o consensualmente) residentes tanto en México como en Estados Unidos son menos propensas a insertarse en una ocupación altamente calificada en comparación con sus pares casados o unidos. No obstante, en México esta dimensión parece ser menos discriminante, ya que los no unidos son sólo 11 por ciento menos propensos que los unidos a emplearse en este tipo de ocupaciones, mientras que entre los no unidos residentes en Estados Unidos dicha probabilidad es 24 por ciento menor que entre los unidos conyugalmente. Estas diferencias en el efecto del estado civil guardan relación con la estructura por edad de la población, pues como señalamos en el apartado anterior, los posgraduados residentes en México son más jóvenes que los que radican en el país vecino del norte. Por tanto, los resultados de los modelos se comprenden en la medida en que a mayor edad, mayor es la propensión que tiene una persona de experimentar la unión conyugal.

En lo referente a la categoría nivel de escolaridad, los resultados obtenidos son los esperados y consistentes con lo señalado en el análisis descriptivo. De su lectura se desprende que, entre mayor es el nivel escolaridad, mayor es la propensión a insertarse en una ocupación altamente calificada. En el caso de la población residente en México, lo que los resultados indican es que el contar con el grado de doctor aumenta en un 59 por ciento la probabilidad de emplearse en una ocupación altamente calificada con respecto a los que sólo cuentan con estudios de maestría. En ese mismo sentido, se puede observar que los mexicanos que residen en Estados Unidos y que tienen ese grado académico presentan una razón de probabilidad 1.6 veces

$80 \frac{\text { SEGUNDO SEMESTRE } 2015}{\text { MIGRACIÓN Y DESARROLLO NÚM. } 25}$ 
mayor que la obtenida por sus pares que poseen un grado profesional y maestría. Como puede apreciarse en los resultados de los modelos estimados, el efecto de contar con estudios de doctorado en la propensión de insertarse en una ocupación de alta calificación es muy superior en Estados Unidos a la reportada por sus connacionales residentes en México. Estos resultados aportan evidencia estadísticamente significativa para suponer que los profesionistas mexicanos con un posgrado que residen en Estados Unidos tienen mayores posibilidades de insertarse laboralmente en ocupaciones altamente calificadas y acordes con su nivel de formación académica que sus pares radicados en México.

El efecto de la variable tipo de carrera opera de forma distinta según país de residencia. Entre los posgraduados radicados en México, quienes se formaron en las áreas de CTIM poseen 22 por ciento menor probabilidad de desempeñarse en ocupaciones altamente calificadas, mientras que en Estados Unidos los que estudiaron un posgrado en CTIM presentan un 26 por ciento de probabilidades de colocarse en este tipo de ocupaciones en comparación con los no cTim. Este hallazgo, aunque sorprende, apoya la hipótesis central de este trabajo en el sentido de que en México existe un desaprovechamiento de la mano de obra altamente calificada. Sin embargo, lo más preocupante es que dicho problema afecta a un subgrupo de los profesionistas formados en carreras y áreas del conocimiento que son fundamentales en los procesos de innovación y desarrollo científico y tecnológico en cualquier país, como son los de cTim, quienes a través de sus conocimientos y habilidades contribuyen a la generación de nuevas patentes, tecnologías e innovación, y al desarrollo empresarial e industrial.

En lo que respecta a las variables lugar de estudio y posesión de la ciudadanía estadounidense, incluidas únicamente en el modelo estimado para la población mexicana con posgrado residente en Estados Unidos, los resultados muestran que el hecho de haber estudiado fuera de ese país disminuye en 32 por ciento la propensión de emplearse en una ocupación altamente calificada. Ello sugiere que, si bien el mercado laboral estadounidense ofrece mejores oportunidades para los profesionistas mexicanos, 


\section{CUADRO 3}

Factores asociados a la probabilidad de que la población con posgrado se inserte en una ocupación altamente calificada en México

(Resultados del modelo de regresión logística), 2010

\begin{tabular}{|c|c|c|c|c|c|c|}
\hline & ODDS RATIO & ERROR STD. & z & $P>Z$ & & AL 95\%] \\
\hline \multicolumn{7}{|l|}{ Sexo } \\
\hline Hombre & 1.000 & & & & & \\
\hline Mujer & 1.125 & 0.029 & 4.500 & 0.000 & 1.069 & 1.184 \\
\hline \multicolumn{7}{|l|}{ Grupo de edad } \\
\hline 40 años o más & 1.000 & & & & & \\
\hline Menor de 40 años & 0.827 & 0.021 & -7.550 & 0.000 & 0.787 & 0.869 \\
\hline \multicolumn{7}{|l|}{ Estado civil } \\
\hline Unido & 1.000 & & & & & \\
\hline No unido & 0.890 & 0.024 & -4.370 & 0.000 & 0.844 & 0.937 \\
\hline \multicolumn{7}{|l|}{ Nivel de escolaridad } \\
\hline Maestría & 1.000 & & & & & \\
\hline Doctorado & 1.595 & 0.067 & 11.150 & 0.000 & 1.469 & 1.732 \\
\hline \multicolumn{7}{|l|}{ Tipo de carrera } \\
\hline \multicolumn{7}{|l|}{ No cTIM } \\
\hline CTIM & 0.785 & 0.023 & -8.360 & 0.000 & 0.742 & 0.831 \\
\hline Constante & 3.055 & 0.070 & 48.670 & 0.000 & 2.921 & 3.196 \\
\hline Número de Obs. & & & & & & 34,598 \\
\hline Prob>chi2 & & & & & & 0.000 \\
\hline Pseudo R2 & & & & & & 0.008 \\
\hline Log likelihood & & & & & & $-19,906.9$ \\
\hline
\end{tabular}

Fuente: Estimaciones de los autores con base en INEGI: Censo de Población y Vivienda de 2010.

un segmento importante de ellos se inserta laboralmente en ocupaciones no acordes con su formación.

En este sentido, la pregunta que queda latente es, żen qué medida y bajo qué condiciones el país de estudio es un factor que incide en la inserción y en las condiciones laborales de los inmigrantes mexicanos en Estados Unidos? Dicho en otras palabras, żimporta el país de formación educativa para lograr una inserción exitosa en el mercado laboral estadounidense? Por último, en cuanto a la condición de tenencia de la ciudadanía estadounidense, se observa que la probabilidad de emplearse en una ocupación altamente calificada es 53 por ciento menor entre los no ciudadanos que en los que cuentan con dicho estatus migratorio. Este resultado es coherente y consistente con el

$82 \frac{\text { SEGUNDO SEMESTRE } 2015}{\text { MIGRACIÓN Y DESARROLLO NÚM. } 25}$ 


\section{CUADRO 4}

Factores asociados a la probabilidad de que los mexicanos con posgrado se inserten en una ocupación altamente calificada en Estados Unidos

(Resultados del modelo de regresión logística), 2009-2011

\begin{tabular}{|c|c|c|c|c|c|c|}
\hline & ODDS RATIO & ERROR STD. & z & $\mathrm{P}>\mathrm{Z}$ & & AL 95\%] \\
\hline \multicolumn{7}{|l|}{ Sexo } \\
\hline Hombre & 1.000 & & & & & \\
\hline Mujer & 1.267 & 0.105 & 2.850 & 0.004 & 1.076 & 1.491 \\
\hline \multicolumn{7}{|l|}{ Grupo de edad } \\
\hline 40 años o más & 1.000 & & & & & \\
\hline Menor de 40 años & 1.669 & 0.138 & 6.190 & 0.000 & 1.419 & 1.962 \\
\hline \multicolumn{7}{|l|}{ Estado civil } \\
\hline Unido & 1.000 & & & & & \\
\hline No unido & 0.761 & 0.064 & -3.260 & 0.001 & 0.645 & 0.897 \\
\hline \multicolumn{7}{|l|}{ Nivel de escolaridad } \\
\hline Maestría & 1.000 & & & & & \\
\hline Profesional & 0.711 & 0.062 & -3.890 & 0.000 & 0.598 & 0.844 \\
\hline Doctorado & 2.626 & 0.427 & 5.940 & 0.000 & 1.909 & 3.611 \\
\hline \multicolumn{7}{|l|}{ Tipo de carrera } \\
\hline No cTIM & 1.000 & & & & & \\
\hline CTIM & 1.261 & 0.114 & 2.570 & 0.010 & 1.057 & 1.505 \\
\hline \multicolumn{7}{|l|}{ Lugar de estudio } \\
\hline Estados Unidos & 1.000 & & & & & \\
\hline México & 0.683 & 0.063 & -4.120 & 0.000 & 0.569 & 0.819 \\
\hline \multicolumn{7}{|c|}{ Ciudadanía estadounidense } \\
\hline Ciudadano & 1.000 & & & & & \\
\hline No ciudadano & 0.470 & 0.042 & -8.380 & 0.000 & 0.394 & 0.561 \\
\hline Constante & 2.155 & 0.195 & 8.490 & 0.000 & 1.805 & 2.573 \\
\hline Número de Obs. & & & & & & 3,036 \\
\hline Prob >chi2 & & & & & & 0.000 \\
\hline Pseudo R2 & & & & & & 0.068 \\
\hline Log likelihood & & & & & & $-1,913.5$ \\
\hline
\end{tabular}

Fuente: Estimaciones de los autores con base en IPUMS: American Community Survey (ACS), 2009-2011.

reportado en otros estudios, en los que se señala que el no contar con la ciudadanía del país receptor restringe las posibilidades de lograr una integración exitosa en el mercado de trabajo.

Estos datos confirman la existencia de una discriminación institucionalizada en donde, entre otros factores de diferenciación social (entre 
los que seguramente se incluye la etnia y la nacionalidad), el lugar de estudios (variable que posiblemente capte también el efecto de tener más experiencia en el origen que en el destino o al menos experiencia en el mercado laboral de acogida) y la tenencia de ciudadanía, son mecanismos de diferenciación claves en el mercado laboral (Bauder, 2012).

\section{Discusión Y CONCLUSIONES}

Un sector fundamental de trabajadores calificados ligado a los procesos de innovación, incremento de la productividad, generación de nuevas ideas, nuevas tecnologías, nuevas empresas e industrias es el de los profesionistas formados en áreas de CTIM. Se trata de un sector clave, tanto para las economías de países en desarrollo como para las de los más industrializados. Sin embargo, han sido estos últimos los que han desplegado intensas y, muchas veces, agresivas políticas de formación y reclutamiento global de recursos humanos formados en estas áreas. Un ejemplo de ello es la reciente Acción Ejecutiva en materia de migración propuesta por el presidente Barack Obama, que incluye como una de sus políticas centrales el fortalecer y ampliar la capacitación laboral de extranjeros egresados de universidades de Estados Unidos en áreas de стім (The White House, 2014). De hecho, en su discurso de presentación de esta iniciativa, Obama subraya explícitamente el interés de su país por atraer y mantener mano de obra altamente calificada, cuando se pregunta:

Are we a nation that educates the world's best and brightest in our universities, only to send them home to create businesses in countries that compete against us? Or are we a nation that encourages them to stay and create jobs here, create businesses here, create industries right here in America? (Obama, 2014).

Contar con los mejores y más brillantes trabajadores calificados del mundo, como alude Obama en su discurso, es sin duda un aspecto que contribuye a elevar la competitividad y las ganancias de las compañías estadounidenses y de la economía de ese país.

$84 \frac{\text { SEGUNDO SEMESTRE } 2015}{\text { MIGRACIÓN Y DESARROLLO NÚM. } 25}$ 
En el caso de México, no obstante el histórico crecimiento de la población de posgraduados durante la última década, los resultados de este estudio confirman la hipótesis de que el trabajo de estos profesionistas mexicanos está devaluado, particularmente entre los trabajadores con posgrado formados en áreas de CTIM. La comparación de las condicionantes laborales de los posgraduados mexicanos en ambos países muestra lo siguiente. En el caso de México: 1) el mercado laboral «castiga» a los posgraduados formados en áreas de CTIM, pues las probabilidades de participar en los segmentos más altos de la estructura ocupacional son significativamente menores a las que presentan los posgraduados formados en otras áreas; 2) de igual forma, el mercado laboral mexicano "castiga» a los jóvenes, en virtud de que las probabilidades de acceder a ocupaciones altamente calificadas son significativamente menores que para los posgraduados mayores de 40 años; 3) pese a que las mujeres con posgrado presentan una probabilidad mayor que los hombres de acceder a ocupaciones altamente calificadas en ambos países, en el mercado de trabajo de Estados Unidos las condiciones son aún más favorables para las mujeres; 4) lo mismo acontece con los posgraduados que poseen estudios de doctorado, en virtud de que la probabilidad de participar en los segmentos más altos de la estructura ocupacional es considerablemente mayor en Estados Unidos que en México.

En el caso de Estados Unidos, los resultados muestran que: 1) el mercado laboral de ese país "premia» a los posgraduados mexicanos formados en áreas de CTIM, cuyas probabilidades de acceder a ocupaciones altamente calificadas son mayores a las de aquellos que se formaron en otras áreas del conocimiento. Exactamente lo opuesto a lo que sucede en México; 2) el mercado laboral estadounidense "premia» a los jóvenes mexicanos, a quienes les ofrece mayores probabilidades de acceder a puestos de trabajo calificados que a los mayores de 40 años. Este resultado también es opuesto a lo que sucede en México; 3) como se había señalado, el mercado laboral estadounidense presenta condiciones laborales más favorables en comparación con México (en cuanto al acceso a ocupaciones de alta calificación), tanto para las mujeres mexicanas con posgrado, como para aquellos que poseen estudios de doctorado; 4) por último, el mercado laboral de Estados Unidos castiga a los 
posgraduados que estudiaron en México, así como a los que no tienen la ciudadanía de ese país.

En suma, el mercado laboral mexicano no es favorable a los posgraduados en áreas de CTIM, a los más jóvenes, y ofrece condiciones menos propicias para las mujeres y para los que poseen estudios de doctorado, en relación con las que brinda Estados Unidos (esto último porque en realidad son mejores para mujeres y doctores). En contraste, el mercado laboral de Estados Unidos "premia» a los posgraduados mexicanos en áreas de CTIM, a los más jóvenes, favorece a las mujeres, a los que cuentan con grado de doctor, a los que estudiaron en aquel país y a los mexicanos que poseen la ciudadanía norteamericana.

La subutilización del trabajo en los países de origen, particularmente entre los trabajadores de CTIM, es un elemento que sin duda está vinculado con su salida masiva hacia otros países. Una lectura de este proceso bajo la lente de la economía neoclásica sugeriría que se trata de una complementariedad de los mercados de trabajo, en el sentido de que las deficiencias presentes en el mercado laboral mexicano pueden ser satisfechas en el estadounidense, estableciéndose así una situación de equilibrio laboral entre ambos países. Sin embargo, en nuestra opinión, se trata de una integración económica y laboral asimétrica, en donde los trabajadores calificados enfrentan una discriminación institucionalizada en el origen, que los lleva a buscar mejores oportunidades laborales en otros países. En este trabajo comparamos las condiciones del mercado laboral de los posgraduados mexicanos tanto en México como en Estados Unidos, y encontramos ciertas condiciones y perfiles laborales que los favorecen más en este último país. Lo cierto es que esta discriminación laboral no es privativa del origen, sino que también se presenta en el destino, al menos en lo que respecta al acceso a un empleo acorde. No obstante, queda por verse en qué condiciones laborales se encuentran ocupados, tema que abordaremos en futuras investigaciones.

Algunas reflexiones de corte metodológico derivadas de la presente investigación apuntan a la necesidad de dimensionar transnacionalmente el análisis de un mercado de trabajo que, en estricto sentido, es transnacional, y entenderlo más allá de lo que pasa en el origen o en el destino. Esto fortalece

$86 \frac{\text { SEGUNDO SEMESTRE } 2015}{\text { MIGRACIÓN Y DESARROLLO NÚM. } 25}$ 
la idea de que es necesario romper con el nacionalismo metodológico en el estudio de los mercados laborales y la movilidad internacional de personas altamente calificadas, así como imprimir una perspectiva más estructural en el análisis del funcionamiento de los mercados de trabajo de esta mano de obra, reconociendo la idea de que la discriminación institucionalizada o la devaluación del trabajo calificado es una condición central de esta integración asimétrica.

\section{Bibliografía}

BAUder, Harald (2003), "Brain Abuse", or the Devaluation of Immigrant Labour in Canada", Antipode, 35, pp. 699-717. (2012), «The International Mobility of Academics: A Labour Market Perspective», International Migration, vol. 35, núm. 1.

BeCKer, Gary S. (1964), Human Capital, Nueva York, Columbia University Press for the National Bureau of Economic Research.

Bourdieu, Pierre (1986), «The Forms of Capital», en J. Richardson (ed.), Handbook of Theory and Research for the Sociology of Education, Nueva York, Greenwood.

Fernández, Christina y A. Carolina Ortega (2008), «Labor Market Assimilation of Immigrants in Spain: Employment at the Expense of Bad Job-matches?», Spanish Economic Review, 10, pp. 83-107.

GandinI, Luciana y Fernando Lozano (2012), «La migración mexicana calificada en perspectiva comparada: el caso de los profesionistas con posgrado en Estados Unidos, 2001-2010», en Telésforo Ramírez y M. Ángel Castillo (coords.), El estado de la migración. México ante los recientes desafios de la migración internacional, México, Conapo, pp. 87-126.

Hernández Laos, Enrique, R. Ricardo Solís y Ana F. Stefanovich H. (2013), Mercado laboral de profesionistas en México. Diagnóstico (2000-2009) y prospectiva (20102020), México, Asociación Nacional de Universidades e Instituciones de Educación Superior (ANUIES).

Hosmer, D. y S. Lemeshow (1989), Applied logistic Regression, Nueva York, Wiley and Sons. 
Instituto Nacional de Estadística, Geografía e Informática (INEGI), Censo de Población $y$ Vivienda 2010, en http://www.inegi.org.mx/est/contenidos/proyectos/ccpv/ default.aspx (consulta: septiembre de 2015).

JAsso, Guillermina, Mark R. Rosenzweig y James P. Smith (2002), "The Earnings of U.S. Immigrants: World Skill Prices, Skill Transferability and Selectivity» (mimeo.).

Langdon, David, George McKittric, David Beede, Beethika Khan y Mark Doms (2011), «STEM: Good Jobs Now and for the Future», U.S. Department of Commerce. Economics and Statistics Administration. http://www.esa.doc.gov/ Reports/stem-good-jobs-now-and-future

Lozano Ascencio, Fernando y Luciana Gandini (2010), Migrantes calificados de América Latina y el Caribe iCapacidades desaprovechadas?, México, Centro Regional de Investigaciones Multidisciplinarias de la UNAM.

Lozano, Fernando y Telésforo Ramírez (en prensa), "Obama’s Immigration Executive Action and STEM workers», Revista Voices of Mexico, CISAn-Unam.

NowickA, Magdalena (2013), "Migrating Skills, Skilled Migrants and Migration Skills: The Influence of Contexts on the Validation of Migrants' Skills», Migration Letters, vol. 11, núm. 2.

MatToo, Aaditya, Ileana Cristina Neagu y Çaglar Özden (2005), «Brain Waste? Educated Immigrants in the U.S. Labor Market», Policy Research Working Paper Series, Washington, D.C., The World Bank, núm. 3581.

Oвама, Barack (2014), «Remarks by the President Barack Obama in Address to the Nation on Immigration", http://www.whitehouse.gov/issues/immigration/ immigration-action\#

Organización Internacional del Trabajo (1998), «Informe I: la medición del subempleo. Decimosexta Conferencia Internacional de Estadísticos del Trabajo, Ginebra, http://www.ilo.org/wcmsp5/groups/public/---dgreports/stat/documents /meetingdocument/wcms_088365.pdf (consulta: 12 de enero de 2015).

Piore, Michael J. (1979), Birds of Passage: Migrant Labor and Industrial Societies, Cambridge, UK, Cambridge University Press.

Salami, B. y Nelson S. (2014), «The Downward Occupational Mobility of Internationally Educated Nurses to Domestic Workers», Nursing Inquiry, 21 (2), pp.153-161.

SIAR, Sheila V. (2013), «From Highly Skilled to Low Skilled: Revisiting the Deskilling of Migrant Labor», Philippine Institute for Development Studies, Discussion Paper Series, núm. 2013-2030.

$88 \frac{\text { SEGUNDO SEMESTRE } 2015}{\text { MIGRACIÓN Y DESARROLLO NÚM. } 25}$ 
U.S. Census Bureau American Community Survey 3-Years Estimates, 2009-2011, en https://usa.ipums.org/usa/ (consulta: agosto de 2014).

The White House (2014), «Immigration Accountability Executive Action», Office of the Press Secretary, http://www.whitehouse.gov/the-press-office/2014/11/20/ fact-sheet-immigration-accountability-executive-action 20 de noviembre de 2014.

Wood, Stephen (1981), The Degradation of Work: Skill, Deskilling and the Braverman Debate, Londres, Harper Collins. 
